\title{
MODEL ORDER REDUCTION OF MIKOTA'S VIBRATION CHAIN INCLUDING DAMPING EFFECTS BY MEANS OF PROPER ORTHOGONAL DECOMPOSITION
}

\author{
WolfGang E. WEBer \\ Chair of Structural Analysis, Helmut-Schmidt-University/University of the Federal Armed Forces Hamburg, \\ Hamburg, Germany; e-mail: wolfgang.weber@hsu-hh.de
}

\section{BERND W. ZASTRAU}

Institute of Mechanics and Shell Structures, Technische Universität Dresden, Dresden, Germany

e-mail: bernd.zastrau@tu-dresden.de

\begin{abstract}
In engineering disciplines, both in scientific and practical applications, systems with a tremendous number of degrees of freedom occur. Hence, there is a need for reducing the computational effort in investigating these systems. If the system behaviour has to be calculated for many time instances and/or load scenarios, the need for efficient calculations further increases. Model order reduction is a common procedure in order to cope with such large systems. The aim of model order reduction is to reduce the (computational) effort in solving the given task while still keeping main features of the respective system. One approach of model order reduction uses the proper orthogonal decomposition. This approach is applied to Mikota's vibration chain, a linear vibration chain with remarkable properties, where two cases of an undamped and a damped structure are investigated.
\end{abstract}

Keywords: Mikota's vibration chain, POD, damping, multibody system, mode shape

\section{Introduction}

Model order reduction (MOR) is a common method in engineering disciplines allowing for efficient calculations of e.g. dynamic behaviour of structures. The basic concept is to reduce the order of the system, for example by reducing the number of degrees of freedom. However, the resulting loss of information should not exceed a certain level. Several techniques for MOR exist. Some of them are physical subspace methods such as Guyan reduction, modal subspace methods or Krylov subspace method (Freund, 2003; Guyan, 1965). While these methods are physically motivated, there are other approaches which do not take physical meaning into consideration. However, these methods still require extraction of main features of the underlying system. One method for this feature extraction is the proper orthogonal decomposition (POD). Once the arbitrary system has been characterised by POD, it is the task of MOR to only take those characteristics into consideration which are needed to adequately describe the (dynamic) behaviour of the system. In this contribution, the model order reduction is applied to a special vibration chain, namely Mikota's vibration chain. Herein, POD is used. Using Mikota's vibration chain has the advantage that its dynamic characteristics have been investigated quite well, such that there exist analytical solutions which serve as reference solutions for the reduced system. Additionally, damping effects are taken into account as some damage phenomena can be modelled by means of damping.

To cope with these tasks, Mikota's vibration chain is introduced in Section 2. A brief description of the approach of model order reduction involving POD is given in Section 3. In Section 4, POD is applied to Mikota's vibration chain. As a next step, a discrete damping element is added 
to Mikota's vibration chain. In doing so, the linearity of the system is kept. POD is then used within MOR to approximate the dynamic behaviour of the damped vibration chain in Section 5 . Finally, conclusions and an outlook are given with Section 6.

\section{Mikota's vibration chain}

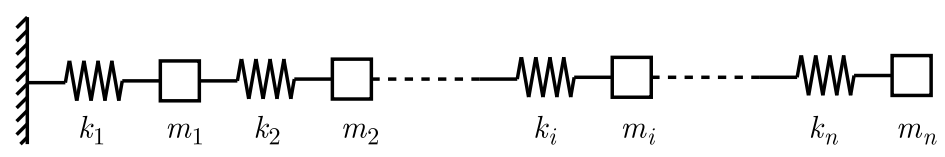

Fig. 1. Undamped vibration chain (Weber et al., 2015)

A linear undamped vibration system with $n$ degrees of freedom (DOF) can be described by

$$
\mathbf{M} \ddot{\mathbf{x}}(t)+\mathbf{K x}(t)=\mathbf{0}
$$

with $\mathbf{x}=\left(x_{1}, \ldots, x_{i}, \ldots, x_{n}\right)^{\mathrm{T}}$ and $\ddot{\mathbf{x}}=\left(\ddot{x}_{1}, \ldots, \ddot{x}_{i}, \ldots, \ddot{x}_{n}\right)^{\mathrm{T}}$ representing the column matrix of displacements and accelerations, respectively. Herein, $\mathbf{M}$ and $\mathbf{K}$ denote the (diagonal) mass and (tri-diagonal) stiffness matrix, respectively. For a linear vibration chain according to Fig. 1 these matrices are

$$
\begin{aligned}
& \mathbf{M}=\operatorname{diag}\left(m_{i}\right) \\
& \mathbf{K}=\operatorname{diag}\left\{\left(k_{1}+k_{2},-k_{2}\right), \ldots,\left(-k_{i}, k_{i}+k_{i+1},-k_{i+1}\right), \ldots,\left(-k_{n}, k_{n}\right)\right\}
\end{aligned}
$$

Mikota set the masses and stiffness coefficients to

$$
m_{i}=\frac{1}{i} m \quad \text { and } \quad k_{i}=(n-i+1) k \quad \text { where } \quad i=1,2, \ldots, n, \quad i \in \mathbb{N}
$$

cf. (Mikota, 2001). Herein, $m$ is the first mass and $k$ denotes the stiffness of the last spring. Mikota conjectured that this specific vibration chain has eigenfrequencies

$$
\Omega_{l}=l \Omega=l \sqrt{\frac{k}{m}} \quad \text { with again } \quad l=1,2, \ldots, n, \quad l \in \mathbb{N}
$$

where $\Omega=\sqrt{k / m}$ is the first eigenfrequency. As can readily be seen, enlarging the system from $n$ DOF to $n+1 \mathrm{DOF}$ changes the mass matrix in such a way that the element $m_{n+1}$ is appended at the lower right corner leading to $\mathbf{M}_{n, n} \rightarrow \mathbf{M}_{n+1, n+1}$, while all other entries of the mass matrix remain the same. In contrast, the corresponding matrix $\mathbf{K}_{n+1, n+1}$ is obtained by adding one row and one column at the upper left corner to the former $\mathbf{K}_{n, n}$. For discussion of this opposite behaviour in the set-up of the matrices and the resulting difficulties for proving Mikota's conjecture to be right, one is referred to e.g., (Müller and Hou, 2007; Müller and Gürgöze, 2006). However, two proofs were proposed by Weber et al. (2015), Müller and Hou (2007). In order to fully describe Mikota's vibration chain also the mode shapes have to be looked at, which was not the focus of Mikota's work. An approach based on polynomial coefficients is given with (Müller and Hou, 2007), a modification of the well-known Laguerre polynomials allowing for evaluating the mode shapes of Mikota's vibration chain is presented in (Weber et al., 2013). But these approaches are quite laborious and do not reveal a structure in order to obtain general formulae for the mode shapes $\mathbf{u}_{l}$. This gap is closed with (Weber et al., 2017), some results of the latter contribution will be used in what follows. 
According to Kochendörffer (1963), the coordinates of the eigenvectors of a matrix - and thus the mode shapes dealt with here - can be expressed by polynomials $p_{l}(i)$ in the coordinate $i$ leading to

$$
\mathbf{u}_{l}=\left(u_{l, i=1}, u_{l, i=2}, \ldots, u_{l, i=n}\right)^{\mathrm{T}}=\left(p_{l}(i=1), p_{l}(i=2), \ldots, p_{l}(i=n)\right)^{\mathrm{T}}
$$

For an arbitrary $n$ DOF and $l \leqslant n$, the first three mode shapes of Mikota's vibration chain are represented by the following polynomials

$$
\begin{aligned}
& p_{l=1}(i)=i \\
& p_{l=2}(i)=i^{2}-\frac{2 n+1}{3} i \\
& p_{l=3}(i)=i^{3}-\frac{3}{5}(2 n+1) i^{2}+\frac{1}{5}\left[\frac{3}{2} n(n+1)+1\right] i
\end{aligned}
$$

see (Weber et al., 2017). There, an approach for determining all $n$ polynomials, i.e. all $n$ mode shapes, in a successive manner is suggested, too. For this special vibration chain it could be proved that the polynomial degree of $p_{l}$ is $l$. Another neat property of the mode shapes of Mikota's vibration chain is the tri-diagonality of the matrix product $\mathbf{U}^{\mathrm{T}} \mathbf{U}$, where $\mathbf{U}$ denotes the modal matrix. It should be noted that, in general, the mode shapes to different eigenfrequencies are not perpendicular to each other with respect to the (standard) scalar product, as only

$$
\mathbf{u}_{l} \mathbf{M} \mathbf{u}_{k}^{\mathrm{T}}\left\{\begin{array} { l l } 
{ = 0 } & { \text { for } \quad l \neq k } \\
{ \neq 0 } & { \text { for } \quad l = k }
\end{array} \quad \mathbf { u } _ { l } \mathbf { K } \mathbf { u } _ { k } ^ { \mathrm { T } } \left\{\begin{array}{ll}
=0 & \text { for } \quad l \neq k \\
\neq 0 & \text { for } \quad l=k
\end{array}\right.\right.
$$

holds.

Exemplary, a graphical representation of the eigenvalues $\lambda_{l}=\Omega_{l}^{2}$ and the first five mode shapes of Mikota's vibration chain with $n=10$ DOF is given in Fig. 2.
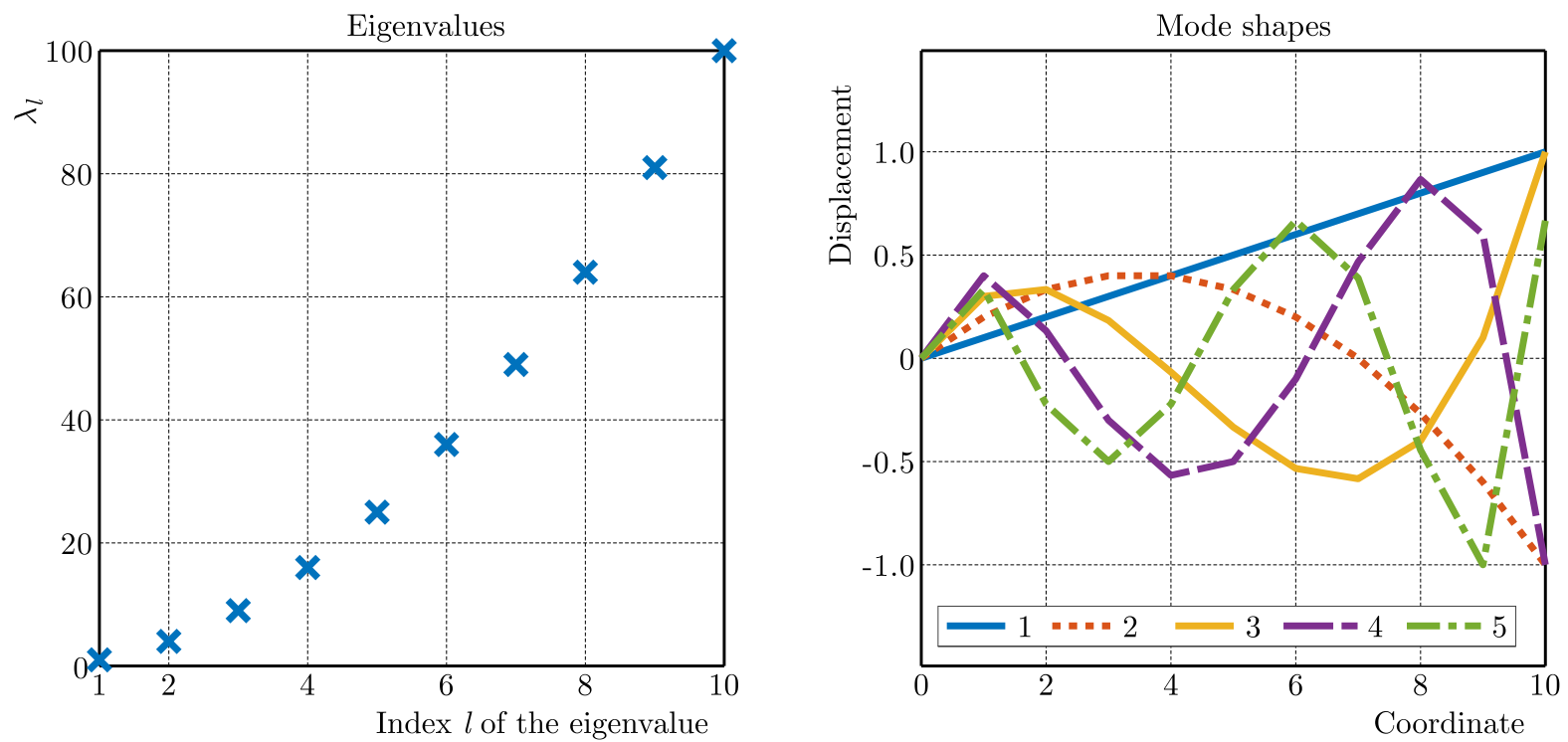

Fig. 2. Eigensolutions of Mikota's vibration chain for $n=10 \mathrm{DOF}$, where only the mode shapes $\mathbf{u}_{l=1}, \ldots, \mathbf{u}_{l=5}$ are shown. The displacements between the coordinates $i$ are interpolated linearly, $i=0$ is at the fixed support according to Fig. 1

\section{A brief introduction to MOR by means of POD}

The solution of typical large systems of equations, which occur in engineering disciplines, requires a huge computational effort. Thus, strategies are sought which allow for reduction of this 
computational cost. One strategy is the model order reduction (MOR). In this strategy, the dimensionality of the underlying mechanical system is reduced while keeping the loss of information within acceptable bounds. For mechanical systems following the system of equations

$$
\mathbf{M} \ddot{\mathbf{x}}+\mathbf{D} \dot{\mathbf{x}}+\mathbf{K x}=\mathbf{f}
$$

with $\mathbf{D}$ denoting the damping matrix, $\dot{\mathbf{x}}$ the vector of velocities and the vector of applied forces $\mathbf{f}$, this order reduction can be performed by projecting the involved vectors from the full space $\mathbb{R}^{n}$ to a lower dimensional space $\mathbb{R}^{n_{\text {red }}}$ using a projection matrix $\boldsymbol{\Phi}$ of dimension $\left(n, n_{\text {red }}\right)$

$$
\mathrm{x} \approx \Phi \mathbf{x}_{\text {red }} \quad \dot{\mathrm{x}} \approx \boldsymbol{\Phi} \dot{\mathbf{x}}_{\text {red }} \quad \ddot{\mathbf{x}} \approx \mathbf{\Phi} \ddot{\mathbf{x}}_{\text {red }}
$$

Inserting these quantities into Eq. (3.1) leads to

$$
\mathbf{M} \Phi \ddot{\mathbf{x}}_{r e d}+\mathbf{D} \boldsymbol{\Phi} \dot{\mathbf{x}}_{r e d}+\mathbf{K} \boldsymbol{\Phi} \mathbf{x}_{r e d}=\mathbf{f}
$$

Multiplying the transpose of the projection matrix from the left finally yields a reduced problem

$$
\begin{aligned}
& \boldsymbol{\Phi}^{\mathrm{T}} \mathbf{M} \boldsymbol{\Phi} \ddot{\mathbf{x}}_{r e d}+\boldsymbol{\Phi}^{\mathrm{T}} \mathbf{D} \boldsymbol{\Phi} \dot{\mathbf{x}}_{r e d}+\boldsymbol{\Phi}^{\mathrm{T}} \mathbf{K} \boldsymbol{\Phi} \mathbf{x}_{r e d}=\boldsymbol{\Phi}^{\mathrm{T}} \mathbf{f} \\
& \mathbf{M}_{r e d} \ddot{\mathbf{x}}_{r e d}+\mathbf{D}_{r e d} \dot{\mathbf{x}}_{r e d}+\mathbf{K}_{r e d} \mathbf{x}_{r e d}=\mathbf{f}_{r e d}
\end{aligned}
$$

The question arises how this projection matrix $\mathbf{\Phi}$ can be obtained. For reducing the dimensionality, the main features of the system have to be extracted. Within this contribution, the proper orthogonal decomposition (POD) is used to extract the main features and thus shall be introduced in what follows.

In the first step, a suitable amount $m$ of observations is necessary. These observations may result from measurements or from analytical or numerical calculations. In the present case, analytical expressions of the displacements will be evaluated numerically and used as observations. The displacement history obtained by the so-called pre-computations is saved in an observation matrix

$$
\mathbf{Q}=\left[\mathbf{x}_{1}, \mathbf{x}_{2}, \ldots, \mathbf{x}_{m}\right]
$$

In general, the number $m$ of observations differs from the number $n$ of DOF and, consequently, $\mathbf{Q}$ is a rectangular matrix. This observation matrix is decomposed by means of the singular value decomposition

$$
\mathbf{Q}=\mathbf{P} \boldsymbol{\Sigma} \mathbf{V}^{\mathrm{T}}
$$

according to Golub and Kahan (1965). Herein, $\mathbf{P}$ denotes the matrix of the left-singular vectors $\phi_{k}$, which will be called proper orthogonal modes (POMs) in what follows. For special cases, these POMs are equivalent to the mode shapes of the respective system. This issue will be addressed in Section 4. The matrix $\boldsymbol{\Sigma}$ is a pseudo-diagonal matrix containing singular values $\sigma_{k}$, with $k \leqslant \min (n, m)$, in a descending order at its main diagonal, whereas all other entries of the rectangular matrix are zero. The matrix $\mathbf{V}$ of the right-singular vectors will not be used within this contribution.

An energy measure for the matrix $\mathbf{Q}$ consisting of a vector sequence is the Frobenius norm, which itself equals the sum of the squared singular values

$$
E_{\text {pseudo }}(\mathbf{Q})=\|\mathbf{Q}\|_{F}^{2}=\frac{1}{m} \sum_{i=1}^{n} \sum_{j=1}^{m} Q_{i, j}^{2}=\sum_{k=1}^{\min (n, m)} \sigma_{k}^{2}
$$

see e.g., (Kerschen and Golinval, 2002). Thus, the value of $\sigma_{k}$ is related to the so-called pseudo-energy associated to the $k$-th POM. As a consequence, one may choose the first $n_{\text {red }}$ POMs, 
with $n_{\text {red }} \ll m$, which capture a certain amount of the system total pseudo-energy. From these POMs, the sought projection matrix

$$
\mathbf{\Phi}=\left[\phi_{1}, \phi_{2}, \ldots, \phi_{n_{r} e d}\right] \quad n_{r e d} \ll m
$$

is constructed.

With this projection matrix, the reduced system according to Eq. (3.4) $\left.)_{2}\right)$ is solved and the results are transferred back to the full system by using Eqs. (3.2). For a detailed review of the POD and some applications the reader is referred to e.g. (Bamer et al., 2017; Fangye et al., 2016; Radermacher and Reese, 2013; Kerschen et al., 2005; Chatterjee, 2000).

\section{Applying POD to Mikota's vibration chain}

As the first step, Mikota's vibration chain is exposed to such an initial displacement which only excites its first mode shape. Afterwards, Mikota's vibration chain performs free vibration. By doing so, the POD should identify the first mode shape only. This is due to the fact that the whole (vibrational) energy of the system is kept in this mode shape. Solving the differential equation with the following parameters

$$
\begin{array}{llll}
m=k=1 & \text { and thus } & \Omega & =\sqrt{\frac{k}{m}}=1 \\
n=10 & t_{0}=0 & \Delta t & =0.01 \quad t_{N}=20 \quad N=2001
\end{array}
$$

where the units have been omitted, yields the displacements $x_{i}$ for each time step $t_{j}$ of $N$. These displacements are written into the observation matrix $\mathbf{Q}$, which is then analysed by means of the POD according to Section 3. All calculations have been performed with MATLAB ${ }^{\circledR}$.

As can be seen in Fig. 3, there is indeed only one (dominant) singular value. The remaining singular values are not of practical relevance, as the quotient $\sigma_{k>1} / \sigma_{1}<10^{-15}$ is in the order of numerical accuracy. Consequently, only the first POM is plotted whereas the remaining POMs are not taken into account. As expected, the POM 1 equals the first mode shape as given by Eq. (2.6) $)_{1}$ and Fig. 2.
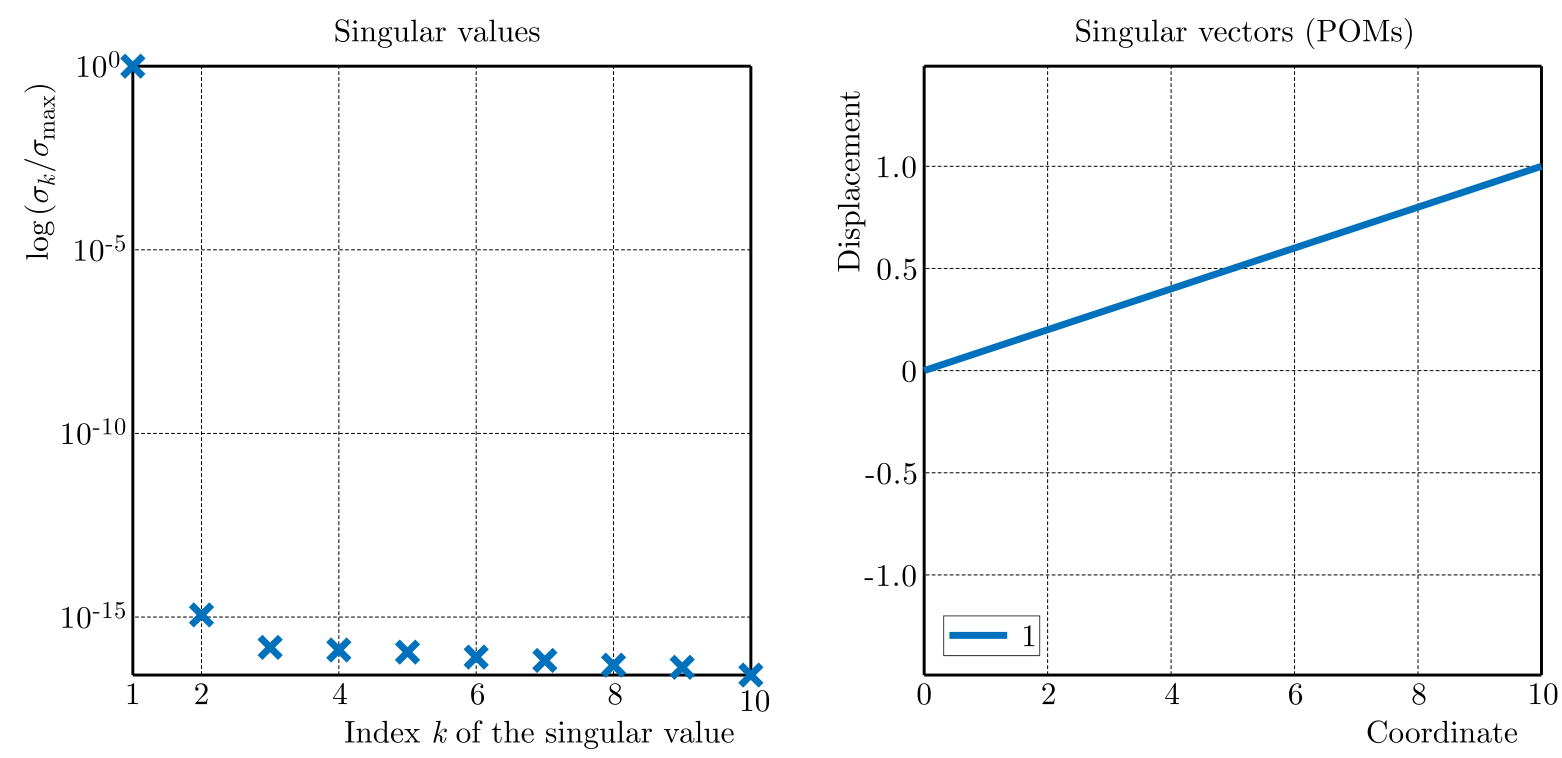

Fig. 3. Singular values and POM of Mikota's vibration chain, $n=10$ DOF and initial excitation of the 1st mode shape, according to the magnitude of $\sigma_{k>1} / \sigma_{1}<10^{-15}$ only the first POM is plotted 
In what follows, Mikota's vibration chain is exposed to a load with a very short time duration, which simulates an impulse or an impact load. In detail, the force

$$
f_{1}(t)= \begin{cases}1 & \text { for } \quad 0 \leqslant t \leqslant 0.05=\delta_{f} \\ 0 & \text { for } \quad \delta_{f}=0.05<t\end{cases}
$$

is applied to the 1st DOF. Compared to the lowest oscillation period (and thus the highest eigenfrequency), the time duration $\delta_{f}$ of the load is small

$$
T_{\text {min }}=\frac{2 \pi}{\Omega_{10}} \hat{=} \frac{2 \pi}{10} \approx 0.628 \gg 0.05=\delta_{f}
$$

with $\Omega_{10}=10 \Omega=10$ according to Eq. (2.4). The resulting displacements and velocities are given with Figs. 4a and 4b, respectively. Some observations from these figures shall be discussed.

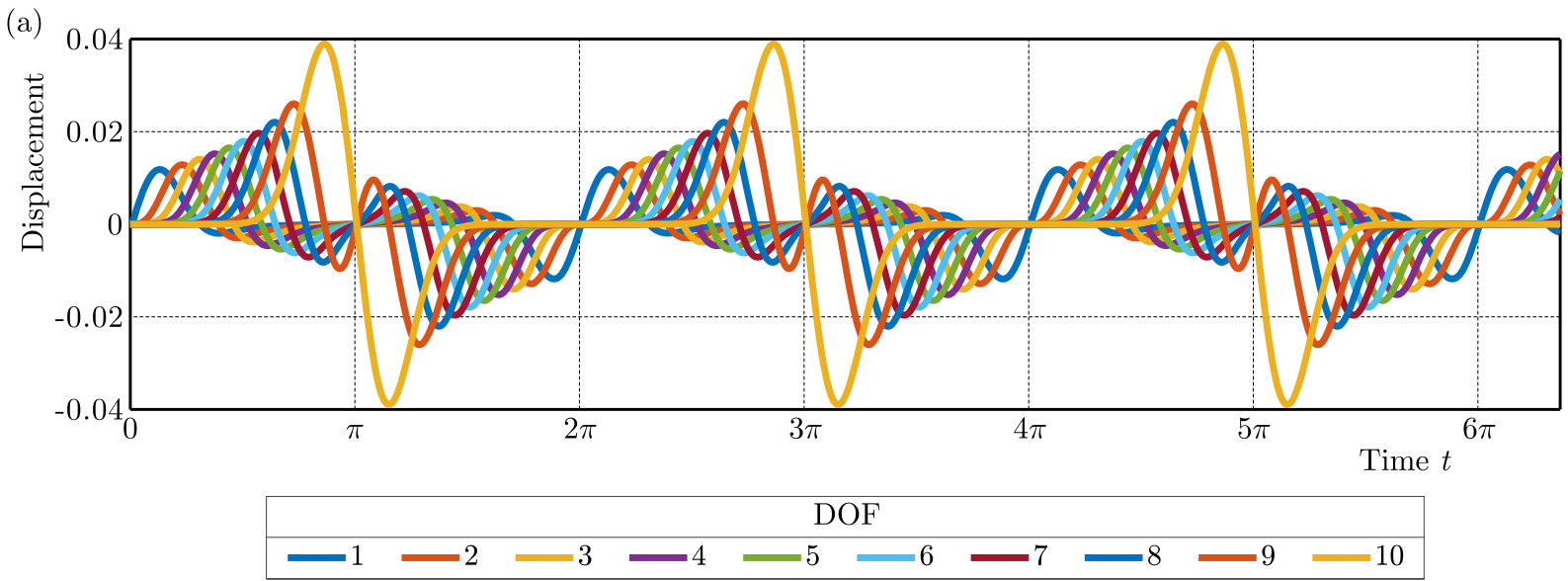

(b)

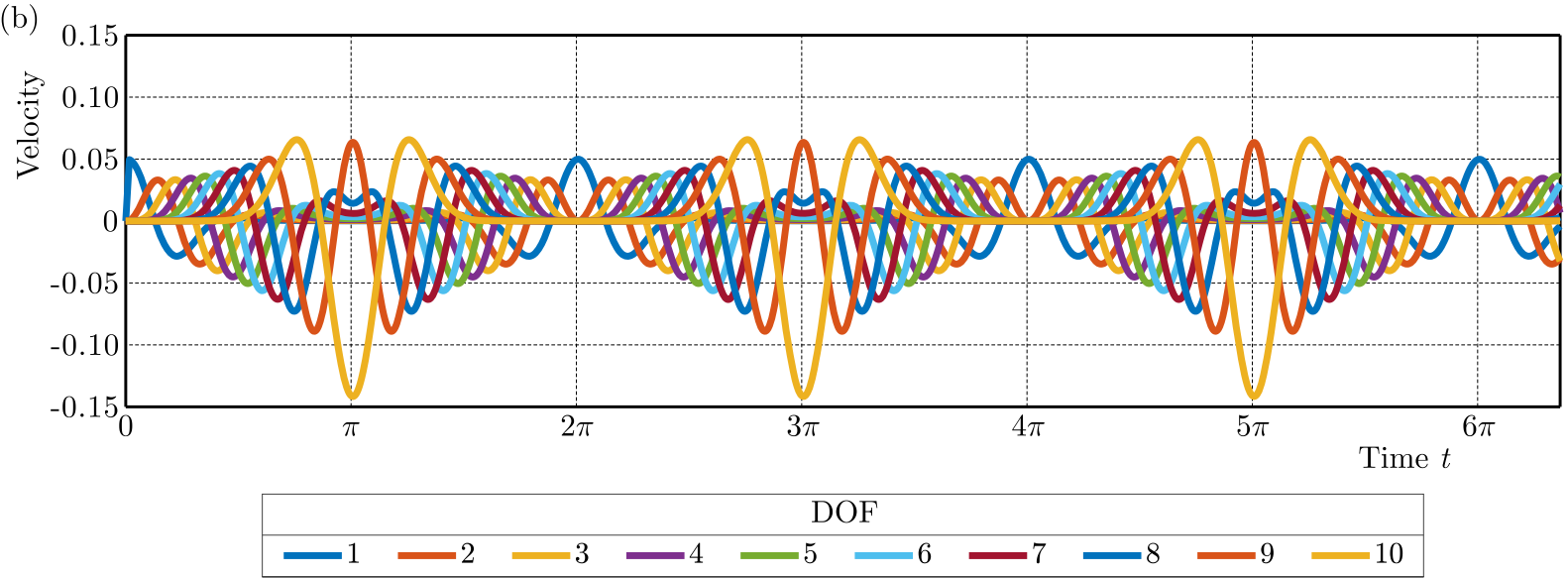

Fig. 4. Displacements and velocities for Mikota's vibration chain with $n=10$ DOF and impulse load according to Eq. (4.3) applied to the 1st DOF

From Fig. 4a, it can be seen that beginning from $t>0$ and starting with the 1st DOF, energy is successively transferred to the remaining DOF. Higher DOFs are characterised by weaker springs and lower masses, see also Eqs. (2.3). The respective displacements increase with the increasing DOF, too. At time instances equal to integer multiples of $\pi$, all DOFs have zero displacements. At time instances equal to integer multiples of $2 \pi$, all DOFs but the 1 st have zero velocities, too. With Eq. (4.4), the impulse load may be regarded as a Dirac-type loading. According to Müller and Schiehlen (1985), such an applied force in fact leads to non-vanishing initial velocities while 
maintaining effectively zero initial displacements. In the present case, the 1st DOF gets an initial velocity (more precisely, the initial velocity is at the time instance $\delta_{f}$ ). At this time instance, the whole system effectively still is at rest, see Fig. 4a. For this reason, the system starts a free oscillation from a state of zero displacement and, consequently, has to return into this state periodically. This happens at the aforementioned time instances at integer multiples of $2 \pi$, where the time duration $\delta_{f}$ has been omitted for clarity. At these time instances, all DOFs but the 1st must have both zero displacements and velocities. The velocity of the 1st DOF equals its initial velocity which is due to the Dirac-type loading, see also Fig. 4b. Due to the special structure of the eigenfrequencies, which is given by Eq. (2.4), the temporal factor at integer multiples of $2 \pi$ of all $n$ mode shapes is identical and equal to 1. Although Fig. 4a may lead to the assumption that there exist pronounced time intervals within which one or more masses $m_{i}$ are at rest, it should be emphasised that this is not the case. On the contrary, there are only distinct time instances - at integer multiples of $\pi$ as discussed above - at which all masses $m_{j}$ have vanishing displacements. All masses but the first are at rest only at integer multiples of $2 \pi$.

The displacements for all time instances according to Eq. (4.2), which are plotted in Fig. 4a, are written in the observation matrix $\mathbf{Q}$, and the latter is investigated by means of POD according to Section 3. The resulting singular values and some POMs are given in Fig. 5.
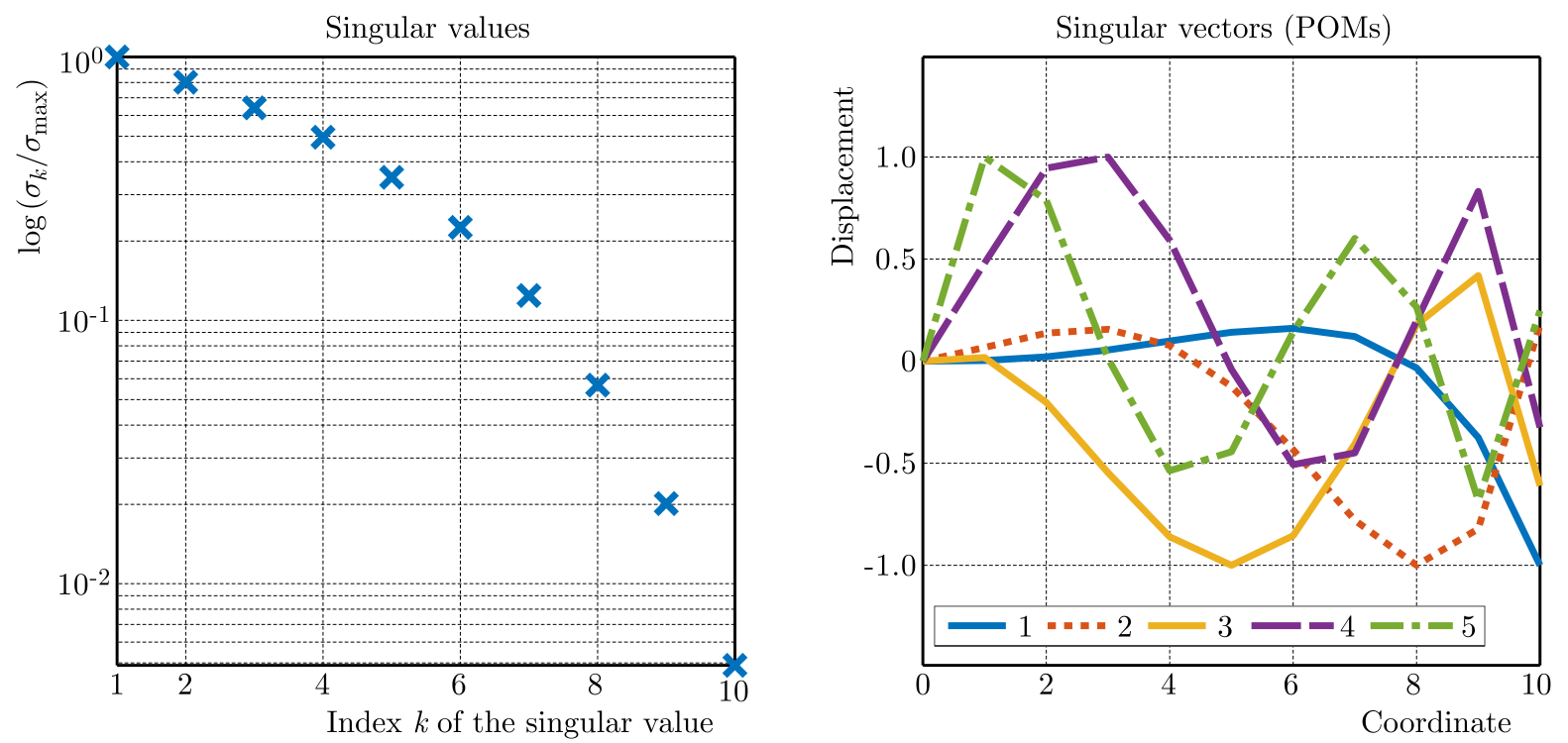

Fig. 5. Singular values and some POMs of Mikota's vibration chain, $n=10$ DOF and impulse load according to Eq. (4.3) applied to the 1 st DOF, only POMs $1, \ldots, 5$ are shown

Compared to Fig. 3, it can readily be seen that in the present load case the so-called pseudo-energy is distributed over all POMs. However, the respective values, i.e. the singular values, are not equal. Hence, the single POMs each have a different contribution to the oscillation pattern of the mechanical system. The descent of the singular values gives an indication of which POMs may be omitted within the model order reduction while not exceeding the given error tolerance. This aspect will be investigated in the next Section, where additionally damping is taken into account.

\section{Applying MOR to modified Mikota's vibration chain including damping}

In this Section, a single absolute damping element is added to Mikota's vibration chain. By doing so, both the eigenfrequencies and mode shapes of the system are changed. For a general introduction to the topic of (optimal) damping, the reader is referred to e.g. (Gürgöze and 
Müller, 1992). For a brief and exemplary discussion concerning the optimal position of absolute and relative damping elements in Mikota's vibration chain, see Weber et al. (2008).

In order to apply a useful model order reduction, the number of DOFs of the system is increased to $n=300$. The absolute damping element is fixed at the 7th DOF. Only one non-vanishing initial excitation $x_{3}=1$ is prescribed at the $3 \mathrm{rd}$ DOF. The other parameters are

$$
m=k=d=1 \quad t_{0}=0 \quad \Delta t=0.01 \quad t_{N}=20 \quad N=2001
$$

where again the units have been omitted. It should be noted that $d=1$ does not lead to weak damping anymore. However, within this contribution, a parameter study is presented. The resulting displacement history for some DOFs is shown in Fig. 6.

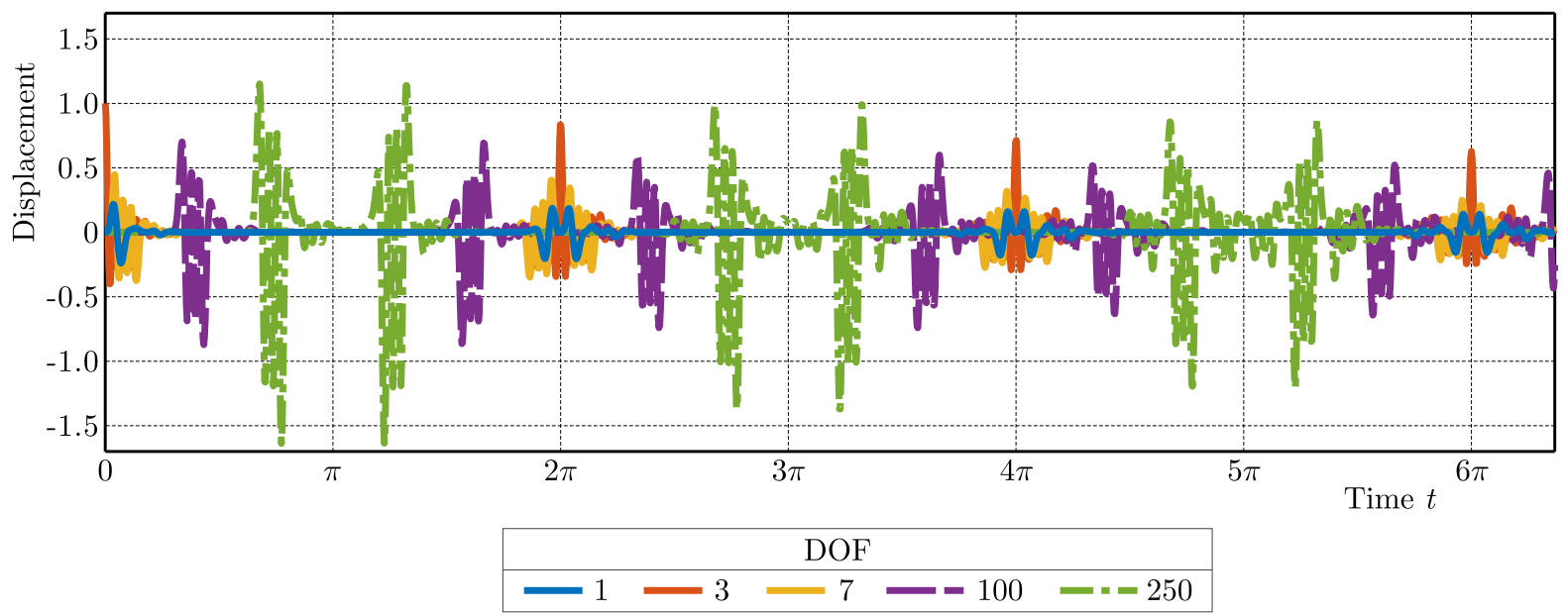

Fig. 6. Displacements for damped Mikota's vibration chain with $n=300$ DOF, initial excitation of the 3rd DOF, absolute damping element at the 7th DOF, only DOF 1,3,7,100,250 are shown

In what follows, the model order reduction using POD is performed. As the initial step, the observation matrix $\mathbf{Q}$ is set by considering the displacements of all $n=300$ DOFs within the time span $t=0$ to $t=10$. The POD is then applied to this observation matrix leading to the singular values and POMs as given in Fig. 7.
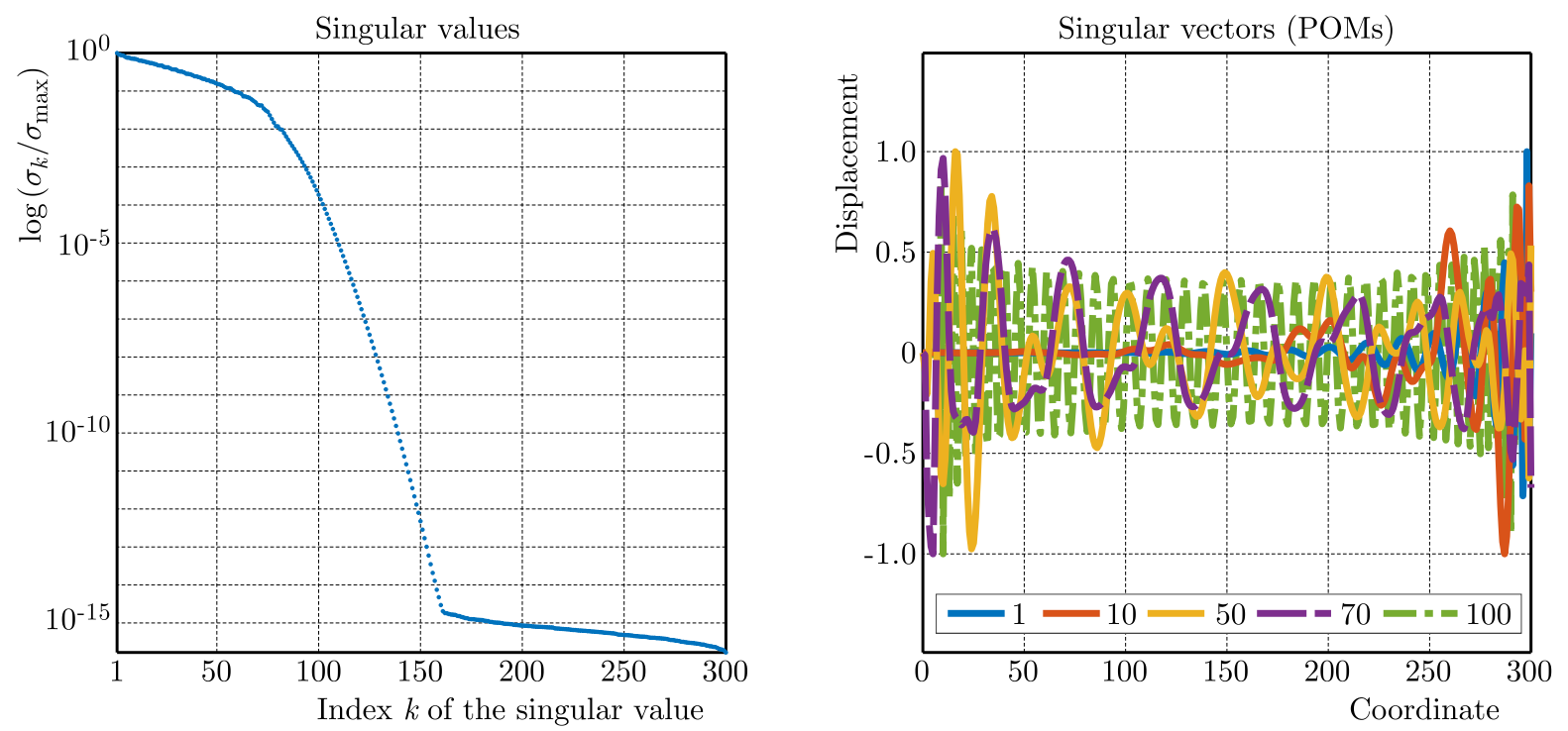

Fig. 7. Singular values and POMs of damped Mikota's vibration chain, $n=300 \mathrm{DOF}$, initial excitation at the 3rd DOF, absolute damping element at the 7th DOF, only POMs 1, 10, 50, 70, 100 are shown 
The so-called pseudo energy $E_{\text {pseudo }}$ of the system can be calculated using Eq. (3.7)

$$
E_{\text {pseudo }}=\sum_{k=1}^{\min (n, m)} \sigma_{k}^{2}=977.5528^{2}
$$

A common approach to the model order reduction is to consider such an amount $n_{\text {red }}$ of POMs that for the corresponding pseudo energy

$$
E_{\text {pseudo,red }} \approx 0.99 E_{\text {pseudo }}
$$

holds (Bamer et al., 2017, Feeny and Kappagantu, 1998). Kerschen et al. (2005) even recommend $E_{\text {pseudo,red }} \approx 0.9999 E_{\text {pseudo }}$. For the present case, the former criterion is fulfilled for $n_{\text {red }}=76$ while the latter criterion gives $n_{\text {red }}=98$.

Thus, in the first step, only 76 POMs are considered. As in the present case $\sigma_{77} / \sigma_{\max }=$ 0.0184 , this means that all POMs, for which $\sigma_{k} \lesssim 0.018 \sigma_{\max }$ holds, are omitted. Although the observation matrix $\mathbf{Q}$ only contains data up to $t=10$, the calculations in the reduced system have been performed until $t=20$. The resulting reduced system is solved and then transferred back to the full system. Both the displacement and velocity history within the time interval $3.25 \pi \leqslant t \leqslant 3.75 \pi$ for the arbitrarily chosen 150 th DOF is given in Fig. 8. Additionally, the diagram contains the displacement and velocity history resulting from calculating the full system. As can be seen, there is a good agreement between these two results for both the displacement and velocity history.
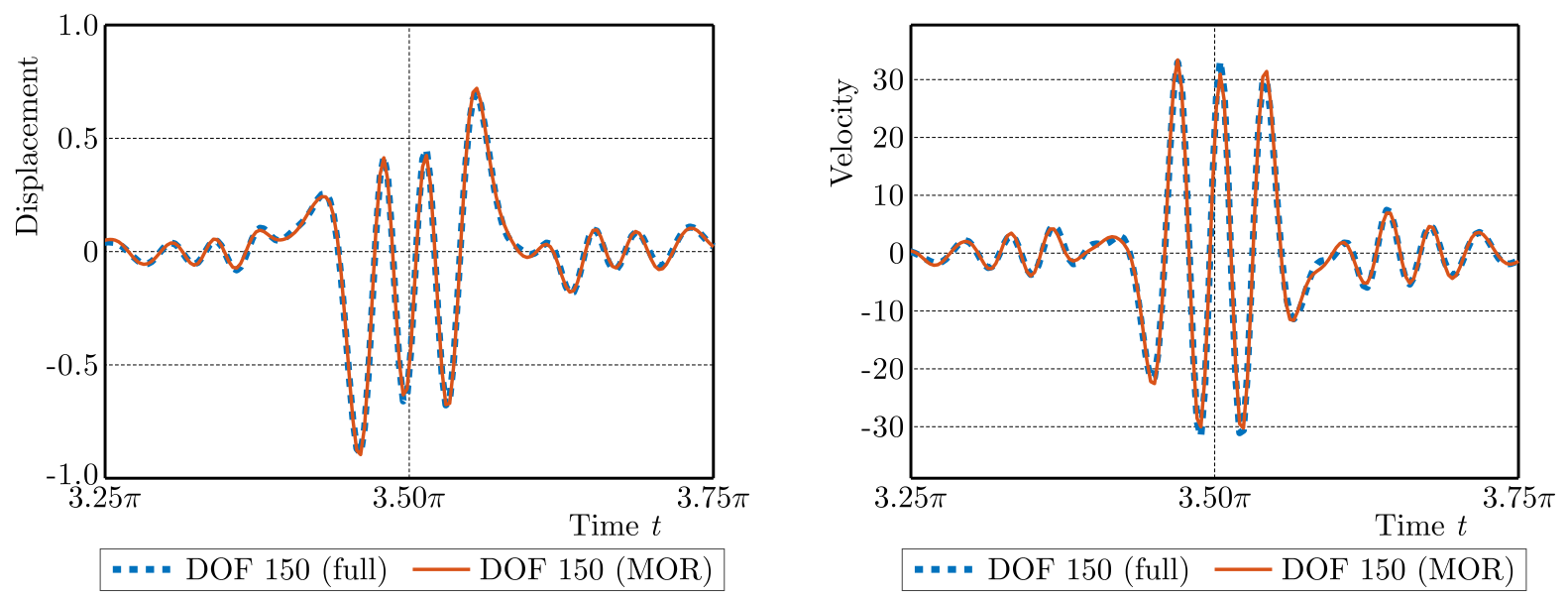

Fig. 8. Displacement and velocity history of the 150th DOF for the full ("full", $n=300$ ) and the reduced ("MOR", $n_{\text {red }}=76$ ) system

For comparison, an additional calculation is performed with $n_{\text {red }}=98$, thus neglecting all POMs for which $\sigma_{k} \lesssim 2.4 \cdot 10^{-4} \sigma_{\max }$. The respective results can be taken from Fig. 9 and do not show any (observable) differences between the results obtained with the full and the reduced system. Besides Eq. (5.3), an additional relation is introduced to measure the deviation between the results obtained with the full system and the results obtained with the reduced system

$$
\Delta E_{\text {phase }}=\sqrt{\frac{\sum_{i=1}^{n}\left[\left(x_{i}-x_{r e d, i}\right)^{2}+\left(\dot{x}_{i}-\dot{x}_{r e d, i}\right)^{2}\right]}{\sum_{i=1}^{n}\left(x_{i}^{2}+\dot{x}_{i}^{2}\right)}}
$$

This relation gives more reliable results as compared to the relation which only takes the pseudo energy into account. This is due to the fact that the latter relation does not reveal pronounced 

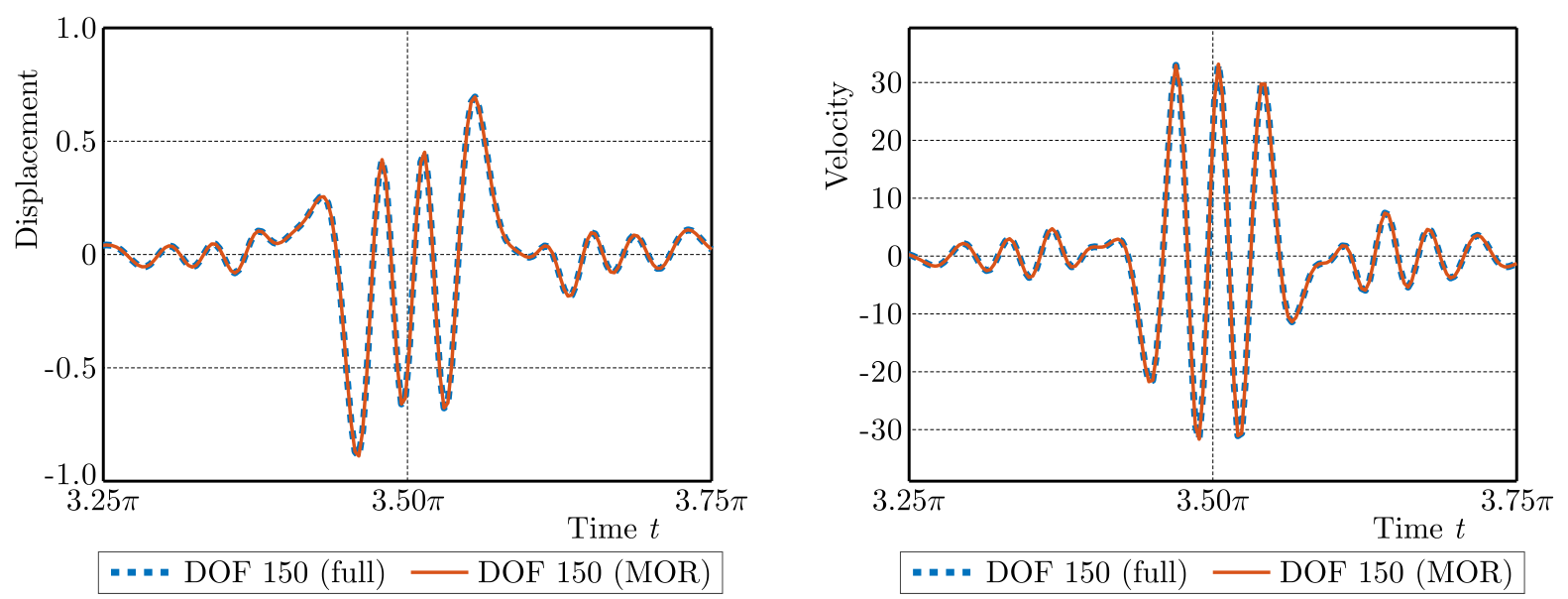

Fig. 9. Displacement and velocity history of the 150th DOF for the full ("full", $n=300$ ) and the reduced ("MOR", $n_{\text {red }}=98$ ) system

phase differences within the displacements and velocities, see also (Kappagantu and Feeny, 1999). In the present case, $\Delta E_{\text {phase }}=0.144$ (compared to 0.5149 for $\left.n_{\text {red }}=76\right)$ thus indicating a very good correlation between the results of the full and the reduced $\left(n_{\text {red }}=98\right)$ system. Hence, the model order reduction has been applied successfully.

\section{Conclusions}

An approach to the model order reduction has been successively applied to Mikota's vibration chain, a special vibration chain having remarkable properties. The chosen approach to the model order reduction involves the proper orthogonal decomposition which, therefore, has been shortly introduced. Some basic insights into the proper orthogonal decomposition were given using the standard (that is, undamped) Mikota's vibration chain with $n=10$ DOFs. Finally, more advanced calculations including the model order reduction were performed after introducing a single absolute damping element into the vibration chain with $n=300$ DOFs. It was observed that the dynamic characteristics of Mikota's vibration chain could be kept if the underlying mechanical system was reduced in such a way, that $>99 \%$ of the so-called pseudo energy was considered. In the present case, an excellent correlation between the results obtained with the full and the reduced system is obtained for $n_{\text {red }}=98$ DOF or - equivalently - for reduction in the dimensionality by $\approx 70 \%$.

In this contribution, a linear vibration system has been investigated. However, non-linear systems play an important role in engineering applications, too. Thus, more scientific work has to be done in the field of model order reduction of non-linear systems. This non-linearity may additionally be caused by damage of the respective structure. Such effects must be taken into account if the model order reduction is used in, e.g., structural health monitoring.

\section{References}

1. Bamer F., Amiri A.K., Bucher C., 2017, A new model order reduction strategy adapted to nonlinear problems in earthquake engineering, Earthquake Engineering and Structural Dynamics, 46, 4, 537-559

2. Chatterjee A., 2000, An introduction to the proper orthogonal decomposition, Current Science, $\mathbf{7 8}, 7,808-818$ 
3. Fangye Y.F., Weber W.E., Zastrau B.W., Balzani D., 2016, Some basic ideas for the simulation of wave propagation in microstructures using proper orthogonal decomposition, Proceedings in Applied Mathematics and Mechanics, 16, 1, 333-334

4. Feeny B.F., Kappagantu R., 1998, On the physical interpretation of proper orthogonal modes in vibrations, Journal of Sound and Vibration, 211, 4, 607-616

5. Freund R.W., 2003, Model reduction methods based on Krylov subspaces, Acta Numerica, 12, $2,267-319$

6. Golub G., Kahan W., 1965, Calculating the singular values and pseudo-inverse of a matrix, Journal of the Society for Industrial and Applied Mathematics, Series B: Numerical Analysis, 2, 2, 205-224

7. Gürgöze M., Müller P.C., 1992, Optimal positioning of dampers in multi-body systems, Journal of Sound and Vibration, 158, 3, 517-530

8. GuYAn R.J., 1965, Reduction of stiffness and mass matrices, AIAA Journal, 3, 2, 380-380

9. Kappagantu R., Feeny B.F., 1999, An "optimal” modal reduction of a system with frictional excitation, Journal of Sound and Vibration, 224, 5, 863-877

10. Kerschen G., Golinval J.-C., 2002, Physical interpretation of the proper orthogonal modes using the singular value decomposition, Journal of Sound and Vibration, 249, 5, 849-865

11. Kerschen G., Golinval J.-C., Vakakis A.F., Bergman L.A., 2005, The method of proper orthogonal decomposition for dynamical characterization and order reduction of mechanical systems: an overview, Nonlinear Dynamics, 41, 1-3, 147-169

12. Kochendörffer R.K., 1963, Determinanten und Matrizen, B.G. Teubner Verlagsgesellschaft, Leipzig

13. Мiкота J., 2001, Frequency tuning of chain structure multibody oscillators to place the natural frequencies at $\Omega_{1}$ and $N-1$ integer multiples $\Omega_{2}, \ldots, \Omega_{N}$, Zeitschrift für Angewandte Mathematik und Mechanik, 81, S2, 201-202

14. Müller P.C., Gürgöze M., 2006, Natural frequencies of a multi-degree-of-freedom vibration system, Proceedings in Applied Mathematics and Mechanics, 6, 1, 319-320

15. Müller P,C., Hou M., 2007, On natural frequencies and eigenmodes of a linear vibration system, Zeitschrift für Angewandte Mathematik und Mechanik, 87, 5, 348-351

16. Müller P.C., Schiehlen W.O., 1985, Linear Vibrations, M. Nijhoff Publishers, Dordrecht

17. Radermacher A., Reese S., 2013, A comparison of projection-based model reduction concepts in the context of nonlinear biomechanics, Archive of Applied Mechanics, 83, 8, 1193-1213

18. Weber W., Anders B., Müller P.C., 2015, A proof on eigenfrequencies of a special linear vibration system, Zeitschrift für Angewandte Mathematik und Mechanik, 95, 5, 519-526

19. Weber W., Anders B., Zastrau B.W., 2008, Some damping characteristics of a chain structured vibration system, Proceedings in Applied Mathematics and Mechanics, 8, 1, 10391-10392

20. Weber W., Anders B., Zastrau B.W., 2013, Calculating the right-eigenvectors of a special vibration chain by means of modified Laguerre polynomials, Journal of Theoretical and Applied Mechanics, Sofia, 43, 4, 17-28

21. Weber W.E., Müller P.C., Anders B., 2017, The remarkable structure of the mode shapes and eigenforces of a special multibody oscillator, Archive of Applied Mechanics, to appear, DOI: $10.1007 /$ s00419-017-1327-9 